\title{
Factors associated with bleeding complications in hernia repair of warfarin users
}

\author{
Ersin Gürkan Dumlu* (D), İbrahim Kılınç² (1), Ömer Parlak ${ }^{1}$, \\ Mustafa Özsoy ${ }^{1}$, Mehmet Kilic ${ }^{3}$
}

\section{SUMMARY}

OBJECTIVE: In this retrospective study, we aimed to determine factors associated with bleeding complications in patients on long-term warfarin, undergoing inguinal hernia repair using low-molecular-weight heparin (LMWH) bridging.

METHODS: Two-year hospital records yielded 44 inguinal hernia repair patients on long-term warfarin (26 men, 4 women, aged 57.4 [38-72] years). All patients were managed with LMWH bridging. Patient and operative characteristics, LMWH bridging characteristics, and international normalized ratio (INR) values were compared between patients with and without postoperative bleeding complications. RESULTS: Indication for warfarin use was heart valve disease $(n=15)$, atrial fibrillation $(n=7)$, deep venous thrombosis $(n=3)$, cerebrovascular event $(n=3)$, and pulmonary embolism $(n=2)$. Four of the operations were urgent, while the remaining were elective. There were four ecchymosis cases and three hematoma cases in a total of seven patients. Baseline $(2.94 \pm 0.26$ versus $2.16 \pm 0.38, p<0.001)$ and preoperative INR values $(1.69 \pm 0.67$ versus $1.31 \pm 0.35, p=0.027)$ were significantly higher, while postoperative INR values $(1.04 \pm 0.09$ versus $1.2 \pm 0.13$, $\mathrm{p}=0.004$ ) were significantly lower in patients having bleeding complications.

CONCLUSIONS: Baseline, preoperative INR, and postoperative INR were the only variables associated with postoperative bleeding complications in patients undergoing LMWH-bridged inguinal hernia repair. We suggest close monitoring of INR levels in long-term warfarin users, even for relatively low-bleeding risk operations such as inguinal hernia repair.

KEYWORDS: Inguinal hernia. Anticoagulants. Warfarin. Low-molecular-weight heparin.

\section{INTRODUCTION}

Inguinal hernia repair is the most common operation in general surgery, with more than 20 million hernia repair operations conducted worldwide, every year ${ }^{1}$. The lifetime risk of having inguinal hernia repair is $27 \%$ for men and $3 \%$ for women ${ }^{2}$. Patients at increased risk of arterial thromboembolism due to conditions, such as atrial fibrillation or prosthetic heart valve, and patients with a history of venous thromboembolism require long-term anticoagulation treatment ${ }^{3}$.

Vitamin $\mathrm{K}$ antagonist warfarin is a commonly used oral anticoagulation agent; however, it may pose a bleeding risk in patients requiring surgery. Warfarin therapy is interrupted in most types of major surgery in order to minimize blood loss. Patients at high risk of a thromboembolic event are recommended to discontinue warfarin and bridge the perioperative period by switching to a short-acting anticoagulant such as subcutaneous low-molecular-weight heparin (LMWH) or intravenous unfractionated heparin ${ }^{4}$. However, guidelines are less clear in patients at moderate risk of bleeding and/or moderate risk of a thromboembolic event. As common as hernia repair may be, the number of studies investigating postoperative complications following LMWH-bridged hernia repair surgery is few.

\footnotetext{
'Ankara Yıldırım Beyazıt University, Faculty of Medicine, Department of General Surgery - Ankara, Turkey.

${ }^{2}$ Ankara Bilkent City Hospital, Department of General Surgery - Ankara, Turkey.

${ }^{3}$ Eskişehir Osmangazi University, Faculty of Medicine, Department of General Surgery - Eskişehir, Turkey.

${ }^{*}$ Corresponding author: gurkandumlu@gmail.com

Conflicts of interest: the authors declare there is no conflicts of interest. Funding: none.

Received on August 23, 2021. Accepted on August 29, 2021.
} 
In this study, we aimed to determine the incidence of bleeding complications and investigate factors related to bleeding complications in long-term warfarin users undergoing inguinal hernia repair via LMWH bridging protocol.

\section{METHODS}

This was a retrospective study of LMWH bridging of patients on long-term warfarin treatment, undergoing inguinal hernia repair. We reviewed files of patients having inguinal hernia repair within the past 2 years and identified 30 patients who had been on long-term warfarin therapy prior to the operation. All patients were managed with LMWH bridging. Patient characteristics, reason for warfarin use, operative characteristics, timing of warfarin discontinuation and reinitiation, timing of LMWH bridging, preoperative and postoperative international normalized ratio (INR) values, and postoperative complications were recorded.

Factors related to complications were investigated. The design of this study has been approved by local Ethics Committees. Due to the retrospective nature of this study, the Ethics Committee did not require written consent from the participants.

\section{Statistical analysis}

SPSS version 17.0 was used in the statistical analysis. Descriptive statistics were used to summarize data: continuous variables were expressed as mean and standard deviation, while categorical variables were expressed as number and percentage. Dependent group comparisons were performed using repeated-measures analysis of variance and paired-sample t test. Independent group comparisons were performed using Mann-Whitney U test. Comparison of categorical variables was performed using chi-square or Fisher's exact test. The limit of statistical significance was set at $\mathrm{p}<0.05$.

\section{RESULTS}

A review of hospital records from the past 2 years revealed 30 inguinal hernia repair patients ( 4 women and 26 men) who had been on long-term warfarin treatment. The mean age of the patients was 57.4 years, ranging between 38 and 72 years. Indication for warfarin use was heart valve disease $(n=15)$, atrial fibrillation ( $\mathrm{n}=7)$, deep venous thrombosis (DVT) $(\mathrm{n}=3)$, cerebrovascular event $(\mathrm{n}=3)$, and pulmonary embolism $(\mathrm{n}=2)$ (Table 1 ). The majority of patients had a primary hernia $(90 \%)$, and most patients were diagnosed with indirect inguinal hernia $(70 \%)$ (Table 1).

Surgical characteristics are summarized in Table 2. Elective surgery was performed on 26 patients, while emergency surgery was performed on 4 patients. In total, 7 (23.3\%) patients had postoperative bleeding complications, with $4(13.3 \%)$ patients having ecchymosis and $3(10 \%)$ developing hematoma. All cases with hematoma resolved naturally without surgical intervention. No venous thromboembolic events were recorded in the postoperative follow-up of patients. There was no significant

Table 1. Patient characteristics.

\begin{tabular}{|c|c|}
\hline & $n(\%)$ \\
\hline Male/female & $26 / 4(86.7 / 13.3)$ \\
\hline \multicolumn{2}{|l|}{ Indication for warfarin use } \\
\hline Heart valve disease & $15(50)$ \\
\hline Arterial fibrillation & $7(23.3)$ \\
\hline Deep venous thrombosis & $3(10)$ \\
\hline Cerebrovascular event & $3(10)$ \\
\hline Pulmonary embolism & $2(6.7)$ \\
\hline \multicolumn{2}{|l|}{ Hernia type } \\
\hline Primary/secondary & $27 / 3(90 / 10)$ \\
\hline Direct & $6(20)$ \\
\hline Indirect & $21(70)$ \\
\hline Femoral & $2(6.7)$ \\
\hline Pantaloon & $1(3.3)$ \\
\hline \multicolumn{2}{|l|}{ Hernia location } \\
\hline Right & $19(63.4)$ \\
\hline Left & $9(30)$ \\
\hline Bilateral & $2(6.6)$ \\
\hline
\end{tabular}

Table 2. Surgical characteristics.

\begin{tabular}{|c|c|}
\hline & $\mathrm{n}(\%)$ \\
\hline Elective surgery & $26(86.7)$ \\
\hline Emergency surgery & $4(13.3)$ \\
\hline \multicolumn{2}{|l|}{ Type of anesthesia } \\
\hline Local & $1(3.3)$ \\
\hline Regional & $22(73.3)$ \\
\hline General & $7(23.4)$ \\
\hline Surgical drain present & $7(23.3)$ \\
\hline$<24 \mathrm{~h}$ & $5(71.4)$ \\
\hline $24-48 h$ & $1(14.2)$ \\
\hline $48-72 \mathrm{~h}$ & $1(14.2)$ \\
\hline Bleeding complications & $7(23.3)$ \\
\hline Hematoma & $4(13.3)$ \\
\hline Ecchymosis & $3(10)$ \\
\hline
\end{tabular}


difference between patients with and without complications in terms of patient or surgical characteristics (Table 3). In addition, the timing of the LMWH bridging protocol was not significantly different between the groups (Table 4). INR readings were taken on the day of warfarin discontinuation (baseline INR), preoperatively on the morning of surgery (pre-op INR), and on the day of warfarin reinitiation (post-op INR).

Baseline (2.94 \pm 0.26 versus $2.16 \pm 0.38, \mathrm{p}<0.001)$ and pre-op INR values $(1.69 \pm 0.67$ versus $1.31 \pm 0.35, \mathrm{p}=0.027)$ were significantly higher, while post-op INR values $(1.04 \pm 0.09$ versus $1.2 \pm 0.13, \mathrm{p}=0.004)$ were significantly lower in patients having bleeding complications (Figure 1).

\section{DISCUSSION}

In this retrospective study, we found the incidence of all bleeding complications was $23.3 \%$, while the incidence of hematoma development was $10 \%$. Baseline, pre-op INR, and post-op INR were the only variables associated with postoperative bleeding complications following LMWH-bridged inguinal hernia repair surgery. Mean INR on the day of surgery was 1.69 in patients developing complications and 1.31 in patients without complications.
Perioperative management of patients on long-term anticoagulation therapy depends on the patient's risk of having a thromboembolic event and the risk of bleeding. However, there are no clear-cut recommendations on perioperative

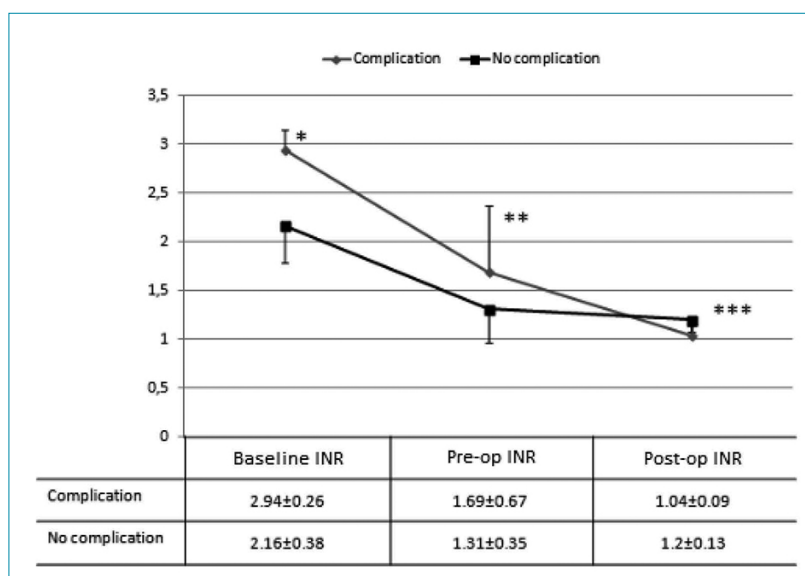

INR: international normalized ratio. ${ }^{*} \mathrm{p}<0.001 ;{ }^{* *} \mathrm{p}=0.027 ;{ }^{* * *} \mathrm{p}=0.004$.

Figure 1. Comparison of international normalized ratio on the day of warfarin discontinuation (baseline INR), on the day of surgery (pre-op INR), and on the day of warfarin reinitiation (post-op INR) in patients with and without bleeding complications.

Table 3. Comparison of patient and surgical characteristics according to complication.

\begin{tabular}{|c|c|c|c|}
\hline & Complication, $n=7$ & No complication, $n=23$ & $\mathrm{p}$ \\
\hline \multicolumn{4}{|l|}{ Gender } \\
\hline Male/female & $6 / 1(85 / 15)$ & 20/3 (87/13) & 1.000 \\
\hline \multicolumn{4}{|l|}{ Hernia location } \\
\hline Right/left/bilateral & $5 / 2 / 0(70 / 30 / 0)$ & $14 / 7 / 2(60 / 3010)$ & 0.667 \\
\hline \multicolumn{4}{|l|}{ Hernia type } \\
\hline Primary/secondary & $6 / 1(85 / 15)$ & $21 / 2(91 / 9)$ & 1.000 \\
\hline \multicolumn{4}{|l|}{ Surgery } \\
\hline Emergency/elective & $0 / 7(0 / 100)$ & $4 / 19(18 / 82)$ & 0.559 \\
\hline \multicolumn{4}{|l|}{ Anesthesia } \\
\hline Local/regional/general & 0/4/3 (0/50/50) & 2/16/5 (8/70/22) & 0.057 \\
\hline \multicolumn{4}{|l|}{ Surgical drain } \\
\hline Yes/no & $3 / 4(43 / 57)$ & $4 / 19(18 / 82)$ & 0.971 \\
\hline
\end{tabular}

Table 4. Comparison of low-molecular-weight heparin bridging periods according to complication.

\begin{tabular}{l|c|c|c} 
& Complication, $n=7$ & No complication, $\mathrm{n}=23$ & $\mathrm{p}$ \\
\hline Warfarin discontinuation and LMWH initiation $^{*}$ & $6.00 \pm 2.61$ & $6.29 \pm 1.71$ & 0.926 \\
\hline Warfarin reinitiation $^{\dagger}$ & $2.67 \pm 0.82$ & $3.14 \pm 0.67$ & 0.138 \\
\hline LMWH discontinuation $^{+}$ & $5.34 \pm 0.52$ & $5.45 \pm 0.61$ & 0.562 \\
\hline
\end{tabular}

LMWH: low-molecular-weight heparin. "Number of days before surgery; ${ }^{\dagger}$ Number of days after surgery. 
anticoagulation management for the majority of patients with a mechanical heart valve, atrial fibrillation, or DVT, who have a moderate risk of thromboembolism and a low/moderate risk of bleeding ${ }^{4}$.

Our standard perioperative anticoagulation management protocol in hernia repair surgery involves warfarin interruption and LMWH bridging. LMWH bridging was shown to be a safe and effective method to manage anticoagulation during the perioperative period of a variety of surgeries, in patients with mechanical heart valves, atrial fibrillation, or DVT in retrospective observational studies and prospective registries ${ }^{5-7}$. LMWH is preferred to unfractionated heparin as it can be safely delivered at fixed doses in an outpatient setting, minimizing hospital stay and its related costs ${ }^{8}$.

The incidence of bleeding complications found in this study was similar to the rates reported by Bombuy et al. ${ }^{9}$, who employed the same standard LMWH bridging protocol for patients on long-term warfarin therapy undergoing elective hernia repair. In their study, among 47 patients bridged with LMWH in the perioperative period, $6(13 \%)$ had surgical-site hematoma and 1 patient had major bleeding, necessitating surgical intervention ${ }^{9}$. No thromboembolic events or deaths were seen in either study.

As hernia repair is considered a low-bleeding risk surgery, some groups investigated the possibility of continuing therapeutic dose warfarin in the perioperative period. In a retrospective study, Sanders et al. ${ }^{10}$ investigated the outcome of inguinal repair surgery in 49 patients while using full-dose warfarin. The rate of small hematomas requiring no intervention was $14.2 \%$, while the rate of large hematomas requiring surgical or medical intervention was $8.2 \%$. They found a significantly higher risk of hematoma in patients with INR $>3$ and suggested that patients may undergo inguinal repair while on warfarin therapy, as long as INR is $<3$. In another study, McLemore et al. ${ }^{11}$ reported a similar rate of postsurgical hematoma in inguinal hernia repair patients who continued warfarin (2/19) and those who had heparin bridging $(2 / 15)$, even though most patients continuing warfarin had an INR $>3$ and those with heparin bridge had INR $<1.5$. However, they indicated that due to a limited number of patients, their study may lack statistical power to detect a significant difference between surgical-site hematomas. Although these studies appear to undermine the importance of having INR $<1.5$ preoperatively, there could be inherent biases in the selection of patients, as warfarin continuation was not their standard protocol.

The American Academy of Neurology published a recent guideline on periprocedural management of antithrombotic medications in patients with ischemic cerebrovascular disease $\mathrm{e}^{12}$, where inguinal herniorrhaphy was listed among procedures that possibly do not increase bleeding risk. Thus, they had a Level $\mathrm{C}$ recommendation to continue warfarin in patients undergoing hernia repair. In contrast, we preferred to exercise caution against the continuation of warfarin during hernia repair, since INR was the only significant factor associated with bleeding in our study. As far as we know there is no increased risk of thromboembolism associated with the use of LMWH bridging, warfarin continuation would only serve to simplify the perioperative management protocol. Warfarin continuation during hernia repair would necessarily mean having INR $>2$ or even $>3$ and may yet show an increased risk of bleeding when practiced in larger numbers.

Smoot et al. ${ }^{13}$ recommended meticulous management of patients requiring warfarin, as chronic warfarin may be a risk factor for postoperative hematoma development in inguinal hernia repair. The recommended current procedure is for warfarin interruption that is omitted for 3 days before elective surgery and then used bridging LMWH for patients with the low/moderate bleeding risk category concomitant high-risk thromboembolism ${ }^{14}$. The biological half-life of warfarin is $36-42 \mathrm{~h}$, which is based on this timing of warfarin interruption. In this process, the INR falls below $2^{15}$. Postprocedure INR $>3$ significantly increases the risk of major bleeding (associated decrease in hemoglobin $\geq 20 \mathrm{~g} / \mathrm{L}$ ) in patients on long-term warfarin ${ }^{16}$. Although the value of INR is more clearly defined for major bleeding, it is difficult to estimate the value for minor bleeding. Although the surgeon feels safe to prevent postoperative bleeding with an INR of $<1.5$ preoperatively, INR $<2$ is acceptable in patients at high risk of thromboembolism. In this study, the difference between the values in this INR range seems to be significant for minor bleeding.

\section{CONCLUSIONS}

This study has limitations. Due to the insufficient number of patients included in this study, we could not obtain a significant INR cutoff value for minor bleeding. Since warfarin continuation during inguinal hernia repair is being discussed as a possibility, studies focused on perioperative management ofhernia repair are needed. On the other hand, the strength of this study is the investigation of LMWH bridging in a homogeneous group of hernia repair patients, while the less number of such patients is a limitation. Future studies with large-scale patient series may be useful to determine the appropriate INR range to prevent minor bleeding in low/moderate-risk groups on long-term warfarin. 
INR was the only factor associated with bleeding complications in patients receiving long-term warfarin therapy, undergoing LMWH-bridged inguinal hernia repair. We suggest close monitoring of INR levels in long-term warfarin users, even for relatively low bleeding risk operations such as inguinal hernia repair.

\section{AUTHORS' CONTRIBUTIONS}

EGD: Conceptualization, Methodology, Writing - original draft, Writing - review \& editing. IK: Data curation. Software. ÖP: Formal analysis. MÖ: Project administration. MK: Investigation, Supervision.

\section{REFERENCES}

1. Kingsnorth $A$, LeBlanc K. Hernias: inguinal and incisional. Lancet. 2003;362(9395):1561-71. https://doi.org/10.1016/ S0140-6736(03)14746-0

2. Primatesta P, Goldacre MJ. Inguinal hernia repair: incidence of elective and emergency surgery, readmission and mortality. Int J Epidemiol. 1996;25(4):835-9. https://doi.org/10.1093/ ije/25.4.835

3. Keeling D, Baglin T, Tait C, Watson H, Perry D, Baglin C, et al. Guidelines on oral anticoagulation with warfarin-fourth edition. Br J Haematol. 2011;154(3):311-24. https://doi. org/10.1111/j.1365-2141.2011.08753.x

4. Douketis JD, Spyropoulos AC, Spencer FA, Mayr M, Jaffer AK, Eckman $\mathrm{MH}$, et al. Perioperative management of antithrombotic therapy: Antithrombotic Therapy and Prevention of Thrombosis, 9th ed: American College of Chest Physicians Evidence-Based Clinical Practice Guidelines. Chest. 2012;141(2 Suppl):e326S350S. https://doi.org/10.1378/chest.11-2298

5. Dunn AS, Spyropoulos AC, Turpie AGG. Bridging therapy in patients on long-term oral anticoagulants who require surgery: the Prospective Peri-operative Enoxaparin Cohort Trial (PROSPECT). J Thromb Haemost. 2007;5(11):2211-8. https://doi.org/10.1111/j.1538-7836.2007.02729.x

6. Hammerstingl C, Tripp C, Schmidt H, von der Recke G, Omran $\mathrm{H}$. Periprocedural bridging therapy with low-molecular-weight heparin in chronically anticoagulated patients with prosthetic mechanical heart valves: experience in 116 patients from the prospective BRAVE registry. J Heart Valve Dis. 2007;16(3):28592. PMID: 17578050

7. Jaffer AK, Ahmed M, Brotman DJ, Bragg L, Seshadri N, Qadeer $M A$, et al. Low-molecular-weight-heparins as periprocedural anticoagulation for patients on long-term warfarin therapy: a standardized bridging therapy protocol. J Thromb Thrombolysis. 2005;20(1):11-6. https://doi.org/10.1007/s11239-005-3120-9

8. Spyropoulos AC, Frost FJ, Hurley JS, Roberts M. Costs and clinical outcomes associated with low-molecular-weight heparin vs unfractionated heparin for perioperative bridging in patients receiving long-term oral anticoagulant therapy. Chest. 2004;125(5):1642-50. https://doi.org/10.1378/chest.125.5.1642

9. Bombuy E, Mans E, Hugué A, Plensa E, Rodriguez L, Prats $M$, et al. Elective inguinal hernioplasty in patients on chronic anticoagulation therapy. Management and outcome. Cir Esp. 2009;86(1):38-42. https://doi.org/10.1016/j. ciresp.2009.02.016

10. Sanders DL, Shahid MK, Ahlijah B, Raitt JE, Kingsnorth AN. Inguinal hernia repair in the anticoagulated patient: a retrospective analysis. Hernia. 2008;12(6):589-92; discussion 667-8. https://doi.org/10.1007/s10029-008-0405-x

11. McLemore EC, Harold KL, Cha SS, Johnson DJ, Fowl RJ. The safety of open inguinal herniorraphy in patients on chronic warfarin therapy. Am J Surg. 2006;192(6):860-4. https://doi. org/10.1016/j.amjsurg.2006.08.058

12. Armstrong MJ, Gronseth G, Anderson DC, Biller J, Cucchiara $B$, Dafer $R$, et al. Summary of evidence-based guideline: periprocedural management of antithrombotic medications in patients with ischemic cerebrovascular disease: report of the Guideline Development Subcommittee of the American Academy of Neurology. Neurology. 2013;80(22):2065-9. https://doi.org/10.1212/WNL.0b013e318294b32d

13. Smoot RL, Oderich GS, Taner CB, Greenlee SM, Larson DR, Cragun EB, et al. Postoperative hematoma following inguinal herniorrhaphy: patient characteristics leading to increased risk. Hernia. 2008;12(3):261-5. https://doi.org/10.1007/s10029007-0313-5

14. Spyropoulos AC, Brohi K, Caprini J, Samama CM, Siegel D, Tafur A, et al. Scientific and Standardization Committee Communication: guidance document on the periprocedural management of patients on chronic oral anticoagulant therapy: recommendations for standardized reporting of procedural/ surgical bleed risk and patient-specific thromboembolic risk. J Thromb Haemost. 2019;17(11):1966-72. https://doi. org/10.1111/jth.14598

15. White RH, McKittrick T, Hutchinson R, Twitchell J. Temporary discontinuation of warfarin therapy: changes in the international normalized ratio. Ann Intern Med. 1995;122(1):40-2. https:// doi.org/10.7326/0003-4819-122-1-199501010-00006

16. Clark NP, Douketis JD, Hasselblad V, Schulman S, Kindzelski $\mathrm{AL}$, Ortel TL, et al. Predictors of perioperative major bleeding in patients who interrupt warfarin for an elective surgery or procedure: analysis of the BRIDGE trial. Am Heart J. 2018;195:108-14. https://doi.org/10.1016/j.ahj.2017.09.015 